\title{
Establishing the Rotenone-Induced Parkinson's Disease Animal Model in Wistar Albino Rats
}

\author{
Gangadhara Swamy ${ }^{1}$ Rajendra Holla² Suresh R. Rao \\ ${ }^{1}$ Department of Anatomy, Subbiah Institute of Medical Sciences and \\ Research Center, Shivamogga, Karnataka, India \\ 2Department of Pharmacology, K.S. Hegde Medical Academy, \\ Mangalore, Karnataka, India \\ J Health Allied Sci Nu 2021;11:158-163.
}

Address for correspondence Suresh R. Rao, PhD, Department of Anatomy, Subbiah Institute of Medical Sciences and Research Center, Shivamogga 577201, Karnataka, India (e-mail: s4suresh@yahoo.co.in).

\begin{abstract}
Objective The aim of this study was to establish the safe and effective dose of rotenone-induced Parkinson's disease (PD) in Wistar albino rat.

Materials and Methods Male Wistar albino rats $(n=6)$ aged between 9 and 11 weeks, weight 200 to $250 \mathrm{~g}$, were selected for the study. Rats were divided into four groups namely, A, B, C, and D; Group A served as control received only isotonic saline, groups $B, C$, and D were administered with rotenone $2,2.5$, and $3 \mathrm{mg} / \mathrm{kg}$ body weight (BW), respectively, with a specialized vehicle through intraperitoneal (IP) route once daily. During the procedure, they were observed for the development of the PD signs such as stooped posture, postural instability, akinesia, bradykinesia, and muscular rigidity. BW and behavioral pattern were recorded before the rotenone introduction and also after the onset of PD signs in them. They were sacrificed when the PD phenotype became debilitating and followed by neurochemical assay for dopamine and antioxidants; histological assay for TH-neuronal density and Lewy bodies were performed in the substantia nigra pars compacta (SNpc) of midbrain.

Results Group C and D animals were developed with the PD signs by the 9th day and

\section{Keywords}

- Parkinson's disease

- rotenone

- immunohisto-

chemistry

- Lewy body and dopamine also there was a significant decrease in the BW noticed in them. Additionally, histological studies revealed the decrease in neuronal density and the presence of Lewy bodies in the dopamine neurons of the SNpc. However, it was also noticed that the group D had shown more mortality rate when compared with the Group $C$.

Conclusion Rotenone with $2.5 \mathrm{mg} / \mathrm{kg}$ BW IP was an ideal dose to develop PD signs in Wistar albino rats model that is a highly reproducible and may offer an excellent tool to establish the new neuroprotective treatment strategies.
\end{abstract}

\section{Introduction}

Parkinson's disease (PD) is a chronic progressive debilitating neurodegenerative condition. Characterized by progressive loss (and even death) of the structure and function of dopaminergic neurons in the substantia nigra pars compacta (SNpc) of midbrain that eventually controls the balance

published online May 18, 2021
DOI https://doi.org/

$10.1055 / \mathrm{s}-0041-1726690$

ISSN $2582-4287$ and movement of the body and with the signs and symptoms such as tremors, stooped posture, postural instability, akinesia, bradykinesia, and muscular rigidity. Thus, it disturbs the routine living style and creates a great burden to an individual. ${ }^{1}$ The disease is named after an English doctor James Parkinson, who described an, in detail, explanation regarding the disease in his book "An Essay on the Shaking
(C) 2021. Nitte (Deemed to be University)

This is an open access article published by Thieme under the terms of the Creative Commons Attribution-NonDerivative-NonCommercial-License, permitting copying and reproduction so long as the original work is given appropriate credit. Contents may not be used for commercial purposes, or adapted, remixed, transformed or built upon. (https://creativecommons.org/licenses/by-nc-nd/4.0/).

Thieme Medical and Scientific Publishers Pvt. Ltd. A-12, 2nd Floor, Sector 2, Noida-201301 UP, India 
Palsy" published in 1817. According to him, incidence of PD increases with age and the symptoms worsen slowly over time. ${ }^{2}$ PD itself is not considered as a fatal disease, though it may reduce life expectancy by a modest amount. It is often said that people die "with" PD rather than "of" the disease. Patients often die of age-related diseases such as cancer, heart disease, or stroke. But the most common cause of death in those with PD is pneumonia, because the disease impairs patients ability to swallow, putting them at risk of inhaling or aspirating food or liquids into their lungs, leading to aspiration pneumonia. ${ }^{3}$ Currently available medications such as Levodopa and anticholinergic alkaloids were temporarily reducing the signs of the disease. ${ }^{4}$ To combat the signs and also prevent the progressiveness of the disease, it is essential to know the in-depth knowledge about the pathophysiology of the disease and clues to the underlying cause of the illness. ${ }^{5}$ In relation to this many reserpine, haloperidol, 1-methyl-4-phenyl-1,2,3,6 tetrahydropyridine, rotenone, and 6-hydroxydopamine (6-OHDA) were used to develop PD animal models in the past. ${ }^{6}$ However, the available models lack to reflect the progressive nature of the illness and its complexity in terms of the extent of pathology, biochemical changes, reproducibility, and the predictive value of neuroprotective interventions is uncertain, and the number of relevant outcome variables that can be assessed is limited. Thus, in the present study, we selected the rotenone that is a relatively a new compound compared with others mentioned above with the different doses in a special vehicle to induce the PD in the Wistar albino rats. Its the developing of treatment protocols or using the different drugs to the same disease depending on the pathogenesis of the disease.

\section{Materials and Methods}

\section{Experimental Animals}

Randomly bred adult Wistar albino rats of 9 to 11 weeks of age weighing between 200 and 250 g from a colony were selected for our experiment. All animals were maintained under standard laboratory conditions, with a constant 12 hours light/dark cycle and controlled temperature (25 \pm $2{ }^{\circ} \mathrm{C}$ ) with access to drinking water and pellet diet ad libitum. ${ }^{7}$

\section{Chemicals}

Rotenone (TCI Chemicals India Pvt. Ltd. Chennai, India) was purchased from Essarkay Chemicals \& Equipment Centre, Mangalore and other chemicals and reagents required for the experiments were purchased from the Sri Durga Laboratory Equipment Supplies, Chilimbi Main Road, Mangalore, Karnataka, India.

\section{Preparation of Rotenone Stock Solution}

Rotenone (50 mg) was weighted in standard weighing machine and dissolved in $1 \mathrm{~mL}$ 100\% di methyl sulfoxide (DMSO) (Sigma-Aldrich Chemicals, St. Louis, Missouri). About $0.1 \mathrm{~mL}$ of DMSO mixture was added to $9.9 \mathrm{~mL}$ olive oil to get a solution of $10 \mathrm{~mL}$, with the final concentration of rotenone $0.5 \mathrm{mg} / \mathrm{mL} .{ }^{8}$ Before starting any new experiment begin, fresh stock solution of rotenone was prepared.

\section{Rotenone Administration to Induce the Parkinson's Disease to Male Wistar Albino Rat}

Adult male Wistar albino rats were selected for the study and divided into four groups, namely, A, B, C, and D with each having six rats per group. Group A serves as control received only isotonic saline intraperitoneal (IP); group B rats were administered $2 \mathrm{mg}$, group C with $2.5 \mathrm{mg}$, and group D with $3 \mathrm{mg} / \mathrm{kg}$ body weight (BW) of rotenone stock solution IP once daily under the standard operating procedure. ${ }^{8}$

All the animals were observed for the BW, behavioral changes for rearing ${ }^{9}$ and postural instability ${ }^{10}$ to assess the neurobehavior, open field test for locomotors activities, ${ }^{11}$ and pole test for the grip strength ${ }^{12}$ before and after the experiment, and twice daily for appearance of Parkinsonian features, including stooped posture, postural instability, bradykinesia, muscular rigidity, and unsteady gait. ${ }^{13}$

When the behavioral phenotype became debilitatinglimiting mobility, feeding or grooming, animals were deeply anesthetized using a combination of ketamine $(70 \mathrm{mg} / \mathrm{kg}$ BW) and medetomidine $(0.5 \mathrm{mg} / \mathrm{kg}$ BW $) .{ }^{14}$ Sufficient volume of blood was collected by the cardiac puncture followed by rapid removed of midbrain from the rest of the brain tissue and bisected midsagittal on the ice piece. One hemisphere was post-fixed in $4 \%$ paraformaldehyde for 7 days and then placed in 30\% sucrose for at least 3 days, until infiltration was complete. The striatum from the other half of the brain was dissected on ice, flash frozen and then stored at $-80^{\circ} \mathrm{C}$ until processed for stereology, Lewy bodies' identification, and neurochemical analysis. ${ }^{8}$

\section{Stereology}

Rats' brain was perfused with $100 \mathrm{~mL}$ of phosphate-buffered saline (PBS) followed by $200 \mathrm{~mL}$ of $4 \%$ paraformaldehyde in PBS. The brains were post fixed in $4 \%$ paraformaldehyde for an additional 4 hours and cryoprotected in 30\% sucrose in $\mathrm{PBS}\left(4^{\circ} \mathrm{C}, 2\right.$ days). Coronal brain sections with $25 \mu \mathrm{m}$ were cut on a freezing microtome. On free-floating sections, immunohistochemistry was performed. The primary antibodies were mouse monoclonal anti-TH antibody (1:200 dilution), rabbit anti-HA (1:500 dilution), rabbit antihuman AADC (1:500 dilution), mouse monoclonal anti-â-gal (1:500 dilution), and mouse monoclonal anti-NeuN (1:200 dilution, Sigma, St. Louis, Missouri, United States). Antibodies were visualized using the $A B C$ reagent (Vector Laboratories, Burlingame, California, United States) and biotinylated secondary antibodies. ${ }^{15}$

\section{Identification of the Lewy Bodies}

The SNpc tissue sections were subjected to endogenous peroxidase quenched with $1 \% \mathrm{H}_{2} \mathrm{O}_{2}$ in PBS, treated with blocking buffer $(0.3 \%$ Triton X100 and 5\% normal chicken serum in PBS for overnight at $4^{\circ} \mathrm{C}$ ). Incubated with $\alpha$-synuclein Lewy body's primary antibody followed by washed with PBS and then incubated with a biotinylated goat antimouse secondary 
antibody. The tissues were then exposed to an avidin-biotin peroxidase complex for 2 hours. The peroxidase activity was visualized by a steady diaminobenzidine solution. All immunoreactions were observed using a compound light microscope and these results were measured using the image analysis J 1.46 software to produce numerical data from our immunohistochemistry ( $\mathrm{IHC}$ ) results, and an image analysis was performed using Image Pro Plus software. ${ }^{16}$

\section{Neurochemistry}

Midbrain tissue was weighed and recorded in a standard weighing machine and placed in a cold PBS and homogenized for estimation of striatal neurotransmitters concentration, dopamine, and acetylcholine by the HPLC method ${ }^{17}$ and antioxidant such as total antioxidant capacity (TAC) and superoxide dismutase (SOD). ${ }^{18}$

\section{Animal Ethics Committee Permission}

This study was performed in a CPCSEA-approved laboratory under registration number 115/1999/CPCSEA following all ethical practices as laid down in the guidelines for animal care. This study has been approved by the Institutional Animal Ethics Committee (IAEC) KSHEMA/IAEC/08/2017.

\section{Statistical Analysis}

All statistical analyses were performed using IBM compatible computers using SPSS Statistics 20 software for paired $t$-test and one-way analysis of variance with a Tukey post hoc test and Mann-Whitney $U$ test where ever it is applicable.

\section{Results}

Wistar albino rats when administered with the rotenone $2.5 \mathrm{mg} / \mathrm{kg}$ BW IP once daily, majority of the animals were developed with PD phenotype signs such as stooped posture, postural instability, bradykinesia, muscular rigidity, and gait disturbances by the 9th day in the rats in the groups $C$ and D. However, an increase in the mortality was found in the group $\mathrm{D}$ animals. There was a significant decrease in the BW noticed in the groups C and D (-Table 1). In group C, there was a significant decrease in behavioral patterns such as rearing, postural stability, locomotor activities, and grip strength noted when compared with the control ( - Fig. 1).

Histological studies had revealed that there was a significant decrease in the dopaminergic neurons (-Figs. 2 and $\mathbf{3}$ ) and also the Lewy bodies were present in the persisting dopaminergic neurons of theSNpc of the midbrain of rats( - Table 2 and - Fig.4).

Dopamine concentration was significantly reduced in the SNpc of midbrain of the group C and D rats. However, in group $B$ animals, a nonsignificant decrease in the dopamine concentration was noticed ( - Fig. 5). Acetylcholine concentration was not affected by the rotenone in the SNpc of midbrain in any of the groups. Significant changes in antioxidants, TAC and SOD, were noticed in the midbrain of groups C and D animals (-Fig. 6).

Table 1 Body weight assessment in the rotenone-induced rats

\begin{tabular}{|l|l|l|}
\hline Groups & Day 1 & Day 10 \\
\hline A & $237.3 \pm 4.32$ & $240.2 \pm 4.53$ \\
\hline B & $238.5 \pm 3.27$ & $231.2 \pm 4.91^{* *}$ \\
\hline C & $239.8 \pm 4.53$ & $231.3 \pm 4.36^{* *}$ \\
\hline D & $241.0 \pm 3.16$ & $226.5 \pm 2.88^{* * *}$ \\
\hline
\end{tabular}

Note: Body weight was assessed in rats $(n=6)$ exposed for rotenone with 2, 2.5 , and $3 \mathrm{mg} / \mathrm{kg}$ BW doses to induce Parkinson's disease. Values are expressed in mean \pm standard deviation. Analysis of variance significance, $\mathrm{F}=10.928 .{ }^{* *}$ indicates $p<0.01 .{ }^{* * *}$ indicates $p<0.001,{ }^{* *}$ and $* * *$ indicates difference from control. There was a significant decrease in the body weight was noticed in the animals of groups B, C, and D when the same are compared with the control group.

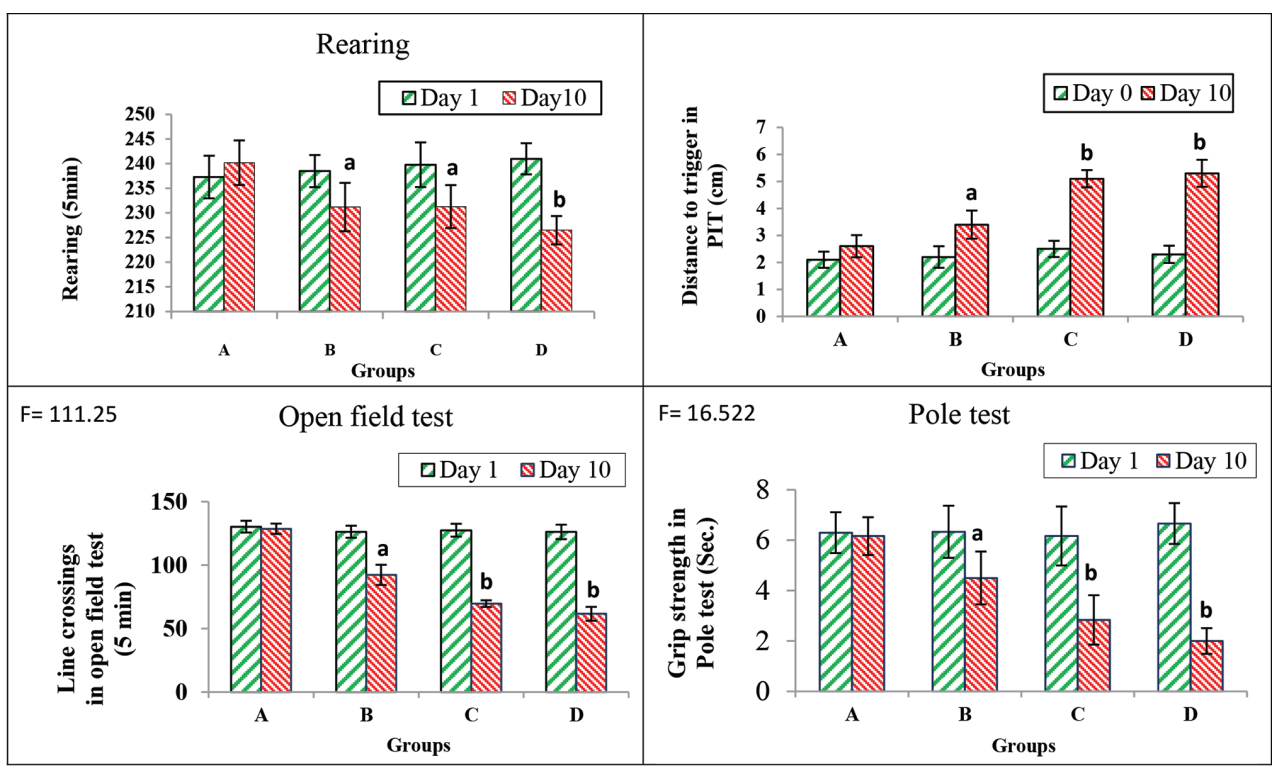

Fig. 1 Behavioral changes were analyzed in rotenone-administered rats $(n=6)$ to induce Parkinson's disease. Values are expressed in mean \pm standard disease. The " $F$ " and " $p$ " values are calculated by one way analysis of variance. aindicates $p<0.05$, bindicates $p<0.001$. 


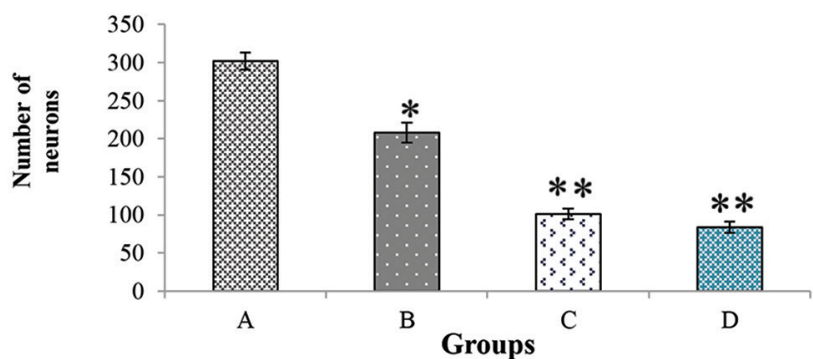

Fig. 2 Tyrosine hydroxylase-neuronal in substantia nigra pars compacta of midbrain in rats exposed to rotenone to induce Parkinson's disease. $a=$ control, $b=$ group $B, c=$ group $C, d=$ group D. Significant decrease in the tyrosine hydroxylase neuronal was noticed in groups $C$ and $D$ animals when the same are compared with the groups $A$ and B (magnification 40X).
Table 2 Lewy bodies count in the SNpc of the mid brain in the rotenone-induced rats

\begin{tabular}{|l|l|}
\hline Groups & Relative Lewy bodies expression \\
\hline A & NIL \\
\hline B & $1.83 \pm 0.75^{*}$ \\
\hline C & $3.67 \pm 1.21^{* *}$ \\
\hline D & $5.33 \pm 1.37^{* *}$ \\
\hline
\end{tabular}

Note: Changes in the relative Lewy bodies expression in substantia nigra pars compacta of midbrain hemisphere in the rats $(n=6)$ on day 10 exposed to rotenone with $2,2.5$, and $3 \mathrm{mg} / \mathrm{kg}$ BW doses to induce Parkinson's disease. Values are expressed in mean \pm standard deviation. Analysis of variance significance, $\mathrm{F}=32.365,{ }^{*}$ indicates $p<0.05,{ }^{* *}$ indicates $p<0.001,{ }^{*}$ indicates difference from control.

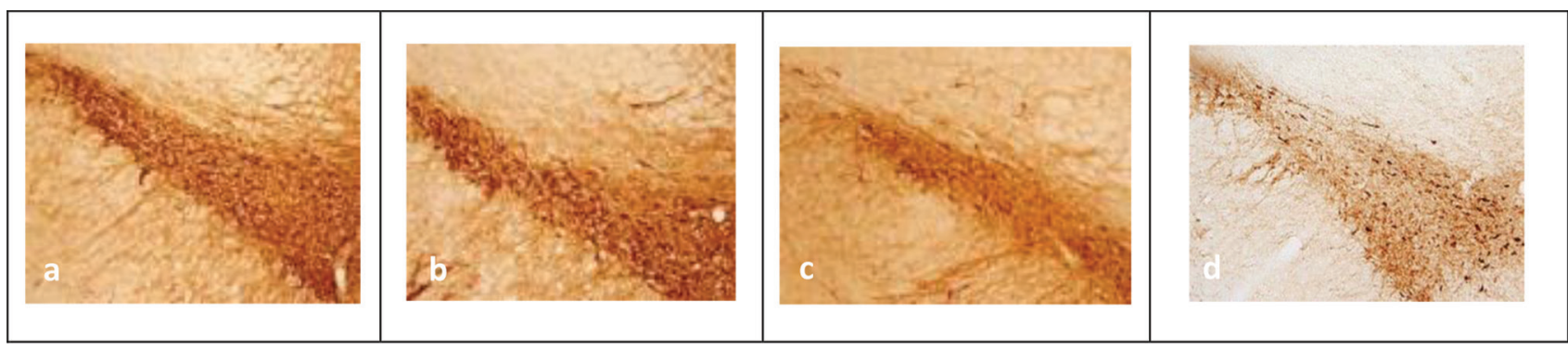

Fig. 3 Tyrosine hydroxylase-neuronal in substantia nigra pars compacta of midbrain in rats exposed to rotenone to induce Parkinson's disease. $\mathrm{a}=$ control, $\mathrm{b}=$ group $\mathrm{B}, \mathrm{C}=$ group $\mathrm{C}, \mathrm{d}=$ group $\mathrm{D}$. Significant decrease in the tyrosine hydroxylase neuronal was noticed in groups $\mathrm{C}$ and $\mathrm{D}$ animals (Magnification 40X).

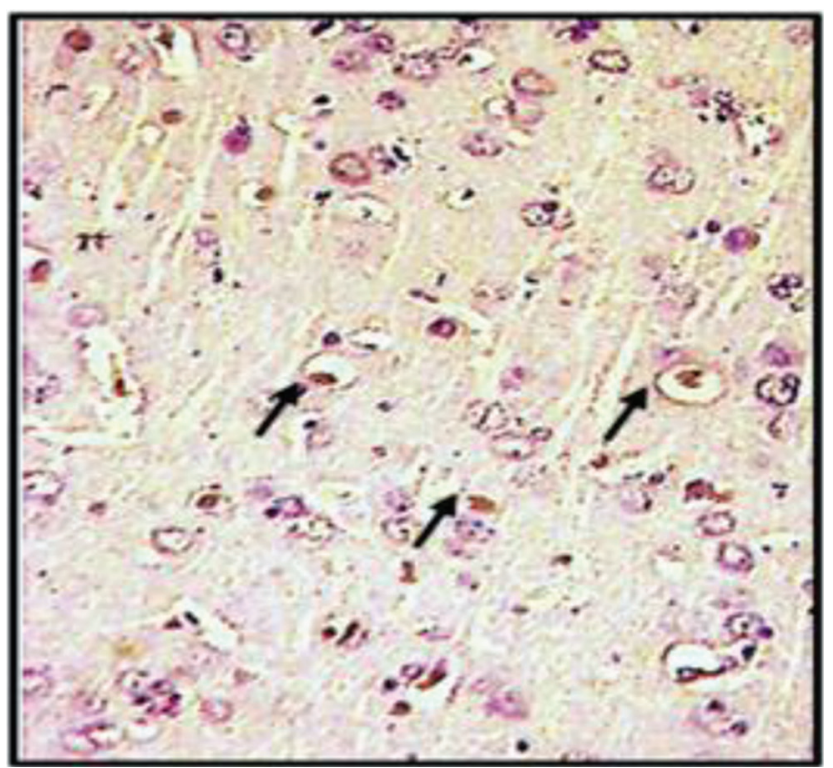

Fig. 4 Lewy bodies in the SNpc in the rotenone-induced rats. Arrow marks show the Lewy bodies in the substantia nigra pars compacta neurons of group $C$ animals exposed to rotenone IP to induce Parkinson's disease.

\section{Discussion}

In our study, it was found that rotenone at $2.5 \mathrm{mg} / \mathrm{kg} \mathrm{BW}$ IP OD administration has induced the PD in Wistar albino rat. Results obtained from BW, behavioral studies, neuronal count, dopamine level, and IHC studies suggest that rotenone

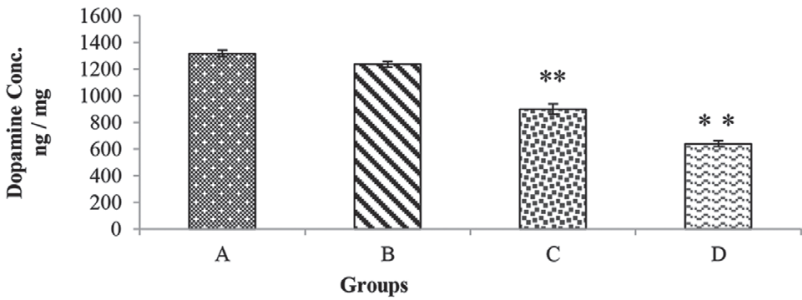

Fig. 5 Dopamine concentration in substantia nigra pars compacta of midbrain hemisphere in the rats $(n=6)$ on day 10 exposed to rotenone to induce Parkinson's disease. Values are expressed in mean \pm standard deviation. Analysis of variance significance, $\mathrm{F}=790.69,{ }^{* *}$ indicates $p<0.001,{ }^{* *}$ indicates difference from control.

below the above said dose is noneffective in inducing the PD and the more than the above said dose is much more toxic and causes the mortality in the animals. Histological studies in the SNpc of rats have shown that PD symptoms do not appear until most nigral neurons are lost, implying that compensatory mechanisms are present and symptoms start only after $70 \%$ of dopaminergic neuronal loss in the SNpc and the earlier studies on the dose of the rotenone similar findings were recorded by the several authors. Alam and Schmidt, 2004, studies using rotenone IP and reported that dopamine depletion and L-dopa-responsive locomotor abnormalities; however, in those studies mortality was high. ${ }^{19}$ Cannon et al ${ }^{20}$ had reported a similar study but they used the rotenone with the lower dose at 2 to $2.5 \mathrm{mg} / \mathrm{kg}$ BW dissolved 2\% DMSO and administered IP route through a special vehicle 98\% Miglyol 


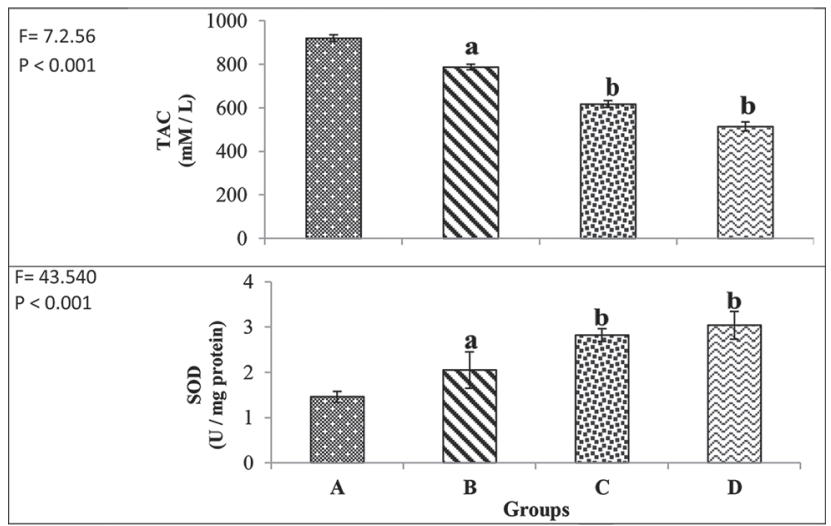

Fig. 6 Antioxidant levels in substantia nigra pars compacta of midbrain tissue homogenate on day 10 in rats $(n=6)$ exposed to rotenone IP to induce Parkinson's disease. Values are expressed in mean \pm $\mathrm{SD}$. The " $\mathrm{F}$ " and " $p$ " values are calculated by one-way analysis of variance. aindicates $p<0.01,{ }^{b}$ indicates $p<0.001$. ${ }^{a}$ Significantly different from control. bSignificantly different from group 2 .

$812 \mathrm{~N}$, had found that rats developed with the PD sings by the 60th day. ${ }^{8}$ In 2018, Mbiydzenyuy et al had administered the rotenone with the same dose but through the subcutaneous route for consecutive 7 to 8 days to induce the PD in the rats. ${ }^{21}$ Deumens et al had developed the similar PD phenotype, but the chemical used to develop the PD was 6-OHDA.22 Betarbet et al described with intravenous and subcutaneous administration of rotenone by osmotic pump, and developed with PD phenotype; but we also found that rotenone through the IP also causes $\alpha$-synuclein accumulation and aggregation in substantia nigra. ${ }^{23}$ Unbiased stereology showed that depletion in the nigral dopamine cell, and it was particularly apparent in the ventral and lateral portions of stratum, the same regions that appear to be most vulnerable in human PD and similar findings were noticed by the Fearnley and Lees in $1991 .{ }^{24}$ In our study we found that rotenone administration results in induction of oxidative stress and neuroinflammation, along with the accumulation of $\alpha$-synuclein positive nigral inclusions, a hallmark of PD. Similar finding were also noticed by Jayaraj et al, in 2019, in there study on rats for the oxidative stress and neuroinflammation in a rat model of PD. ${ }^{25}$ While the concentrations of both acetylcholine and dopamine decrease, the equilibrium between these two neurotransmitters changes to favor acetylcholine. Subsequent release of dopamine from remaining axon terminals pushes an increase of acetylcholine, worsening the imbalance between both neurotransmitters that cause the dyskinesia. Furthermore, in the regimen described here, we found intraneuronal, cytoplasmic inclusions containing $\alpha$-synuclein that have morphology very similar to human Lewy bodies. As noted above, this feature of the model, together with other relevant end points, such as behavioral changes, oxidative damage, changes in the dopamine concentration and stereology makes the rotenone model particularly attractive for studying disease-modifying therapies. Hence, the present study suggests that rotenone at $2.5 \mathrm{mg} / \mathrm{kg}$ BW can be used to develop the PD rat model.

\section{Summary and Conclusion}

Rotenone was injected through the IP route once daily in the Wistar albino rats. By the 9th day, PD signs starts appearing in them. All the animals were included for the BW, behavioral assays. The histopathological and neurochemical features of PD were confirmed by assessing the SNpc for the microscopic and biochemical investigations respectively. Thus, rotenone with $2.5 \mathrm{mg} / \mathrm{kg}$ BW was considered as an ideal dose to induce the PD in the Wistar albino rats.

\section{Conflict of Interest}

None declared.

\section{References}

1 Braak H, Braak E. Pathoanatomy of Parkinson's disease. J Neurol 2000;247(2, Suppl 2):II3-II10

2 Parkinson J. An essay on the shaking palsy. 1817. J Neuropsychiatry Clin Neurosci 2002;14(2):223-236

3 Chang YP, Yang CJ, Hu KF, et al. Risk factors for pneumonia among patients with Parkinson's disease: a Taiwan nationwide population-based study. Neuropsychiatr Dis Treat 2016;12:1037-1046

4 Guridi J, Lozano AM, Lozano AM. A brief history of pallidotomy. Neurosurgery 1997;41(5):1169-1180, discussion 1180-1183

5 Goldman SM. Environmental toxins and Parkinson's disease. Annu Rev Pharmacol Toxicol 2014;54:141-164

6 Marey-Semper I, Gelman M, Lévi-Strauss M. A selective toxicity toward cultured mesencephalic dopaminergic neurons is induced by the synergistic effects of energetic metabolism impairment and NMDA receptor activation. J Neurosci 1995;15(9):5912-5918

7 Pritchett-Corning KR, Chang FT, Festing MF. Breeding and housing laboratory rats and mice in the same room does not affect the growth or reproduction of either species. J Am Assoc Lab Anim Sci 2009;48(5):492-498

8 Cannon JR, Tapias V, Na HM, Honick AS, Drolet RE, Greenamyre JT. A highly reproducible rotenone model of Parkinson's disease. Neurobiol Dis 2009;34(2):279-290

9 Fleming SM, Zhu C, Fernagut PO, et al. Behavioral and immunohistochemical effects of chronic intravenous and subcutaneous infusions of varying doses of rotenone. Exp Neurol 2004;187(2):418-429

10 Woodlee MT, Kane JR, Chang J, Cormack LK, Schallert T. Enhanced function in the good forelimb of hemi-Parkinson rats: compensatory adaptation for contralateral postural instability? Exp Neurol 2008;211(2):511-517

11 Devi PU, Suresh R, Hande MP. Effect of fetal exposure to ultrasound on the behavior of the adult mouse. Radiat Res 1995;141(3):314-317

12 Ogata A, Tashiro K, Nukuzuma S, Nagashima K, Hall WW. A rat model of Parkinson's disease induced by Japanese encephalitis virus. J Neurovirol 1997;3(2):141-147

13 Park JH, Kang YJ, Horak FB. What is wrong with balance in Parkinson's disease? J Mov Disord 2015;8(3):109-114

14 Nair-Roberts RG, Chatelain-Badie SD, Benson E, White-Cooper H, Bolam JP, Ungless MA. Stereological estimates of dopaminergic, GABAergic and glutamatergic neurons in the ventral tegmental area, substantia nigra and retrorubral field in the rat. Neuroscience 2008;152(4):1024-1031

15 Sun M, Zhang GR, Kong L, et al. Correction of a rat model of Parkinson's disease by coexpression of tyrosine hydroxylase and aromatic amino acid decarboxylase from a helper virus-free herpes simplex virus type 1 vector. Hum Gene Ther 2003;14(5):415-424 
16 Gao C, Wang AY, Han YJ. Microwave antigen retrieval blocks endogenous peroxidase activity in immunohistochemistry. Appl Immunohistochem Mol Morphol 2008;16(4):393-399

17 Wojnicz A, Ortiz JA, Casas AI, Freitas AE, López MG, Ruiz-Nuño A. Simultaneous determination of 8 neurotransmitters and their metabolites using liquid chromatography in tandem mass spectrometry application to the murine Nrf2 model of depression. Clin Chim Acta 2016;7:714-720

18 Marttila RJ, Lorentz H, Rinne UK. Oxygen toxicity protecting enzymes in Parkinson's disease. Increase of superoxide dismutase-like activity in the substantia nigra and basal nucleus. J Neurol Sci 1988;86(2-3):321-331

19 Alam M, Schmidt WJ. Rotenone destroys dopaminergic neurons and induces parkinsonian symptoms in rats. Behav Brain Res 2002;136(1):317-324

20 Cannon JR, Tapias V, Na HM, Honick AS, Drolet RE, Greenamyre JT. A highly reproducible rotenone model of Parkinson's disease. Neurobiol Dis.2009;34(2):279-290
21 Mbiydzenyuy NE, Ninsiima HI, Valladares MB, Pieme CA. Zinc and linoleic acid pre-treatment attenuates biochemical and histological changes in the midbrain of rats with rotenone-induced Parkinsonism. BMC Neurosci 2018;19(1):29

22 Deumens R, Blokland A, Prickaerts J. Modeling Parkinson's disease in rats: an evaluation of 6-OHDA lesions of the nigrostriatal pathway. Exp Neurol 2002;175(2):303-317

23 Betarbet R, Canet-Aviles RM, Sherer TB, et al. Intersecting pathways to neurodegeneration in Parkinson's disease: effects of the pesticide rotenone on DJ-1, alpha-synuclein, and the ubiquitin-proteasome system. Neurobiol Dis 2006;22(2):404-420

24 Fearnley JM, Lees AJ. Ageing and Parkinson's disease: substantia nigra regional selectivity. Brain 1991;114(Pt 5) :2283-2301

25 Jayaraj RL, Beiram R, Azimullah S, et al. Lycopodium attenuates loss of dopaminergic neurons by suppressing oxidative stress and neuroinflammation in a rat model of Parkinson's disease. Molecules 2019;24(11):2182 